\title{
VoIP over WLANs by Adapting Transmitting Interval and Call Admission Control
}

\author{
Zhuo Chen, Lingyun Wang, Feng Zhang, Xinbing Wang and Wen Chen \\ School of Electronic, Information and Electrical Engineering \\ Shanghai Jiao Tong University \\ Email: \{chenzhuo, wanglingyun, fzhang,xwang8, wenchen\}@ sjtu.edu.cn
}

\begin{abstract}
VoIP over wireless local area network (WLAN) is an important application of WLAN and gaining more and more attention recently. In this paper, we analyze the maximum number of VoIP calls in WLAN and propose a new call admission control strategy, namely, adaptive transmitting interval call admission control (ATICAC) to enhance VoIP calls in 802.11 WLANs. In ATICAC strategy, Base Station (BS) adaptively changes the transmitting interval of the active stations to prevent the network from saturation by controlling the average collision probability $p_{c}$ of the network. The proposed model is in good agreement with our simulation results. ATICAC can not only ensure the QoS of VoIP calls in 802.11 WLANS in the network, but also increase the number of VoIP calls in the network.
\end{abstract}

\section{INTRODUCTION}

Voice over IP (VoIP) has been widely used these years for its simplified infrastructure and significant cost savings. Using VoIP on the network, there is no need to separate cabling for telephone system and the phone can be taken when offices move with the same telephone number. It is because a VoIP exchange is based on software rather than hardware. It is easier to alter as well as maintain with operating costs lowered by $20 \%-30 \%$.

With the diminishing cost of electronic hardware, IEEE 802.11 based wireless local area networks (WLANs) have been massively deployed in public and residential places such as classrooms, airports, and apartments, and more and more devices and peripherals are integrated with WLAN access capability. Due to these great developments, in recent years, there have been greatly increasing interests in VoIP in WLANs, in which the IEEE 802.11 distributed coordination function protocol or enhanced DCF protocol is used. However, many challenges still remain in voice over WLAN (VoWLAN). It is well known that widely deployed IEEE 802.11 WLANs employ a contention-based medium access control (MAC) protocol, the distributed coordination function (DCF). Although DCF can well support the best effort traffic, it may introduce arbitrarily large delay and delay jitters; thus, it is unsuitable for real-time applications with strict QoS requirements. In addition, unlike cellular networks where dedicated channels are assigned to voice traffic, voice packets in WLANs are multiplexed with data traffic. When data traffic load increases, the QoS of VoWLAN would be severely degraded. It is

\footnotetext{
${ }^{1}$ This work is supported by NSF China (No. 60702046).
}

a challenging task to provide QoS for voice traffic while maintaining as high throughput as possible for data traffic.

We have not yet well understood the question of how the IEEE 802.11 WLAN can support QoS of VoIP. In this paper, we endeavor to address this problem through both analysis and simulations in Section III. We develop adaptive transmitting interval call admission control (ATICAC) strategy to ensure QoS of VoIP in 802.11 WLANS for supporting major QoS of VoIP metrics, i.e., throughput, delay, and packet loss rate. Our ATICAC strategy can also support more calls in the network compared with normal call admission control in Section IV. Our simulations in Section V demonstrate the effectiveness of our analytical model and show the performance of ATICAC strategy.

\section{RELATED WORK}

Bianchi uses a Markov process to model DCF and evaluates the channel throughput, and frame loss as a function of the number of wireless stations in [6]. Initial studies on the performance of real-time applications over 802.11 are presented by authors in [2], [5]. The authors study the inherent limitations of the $802.11 \mathrm{a} / \mathrm{b}$ DCF in supporting VoIP calls over a WLAN in [16].

Current research works ( [8] and references therein) and the enhanced DCF (EDCF) defined in the IEEE 802.11e draft [9], [10] tend to provide differentiated service rather than stringent QoS assurance. Analysis of interference model in wireless mesh network is made by [1], and gives a call admission control with interference capacity. Several performance optimization schemes are proposed for WLANs to improve the VoIP quality such as in [13], [14], [15]. Authors in [13] propose the use of dual queue of 802.11 MAC to provide priority to VoIP while [14] proposes packet aggregation to increase capacity and [15] proposes an adaptive transmission algorithm over an IEEE 802.11 WLAN that supports integrated voice and data services, where data traffic is transmitted with DCF, while voice transmission is carried out with PCF, and similar approach can be applied to sensor networks as well [11], [12].

\section{Analytical Study of VoIP In The IEEE 802.11 WLANS}

This section focuses on the analysis of the performance of VoIP in the IEEE 802.11 DCF. Note that in the following analysis, the hidden terminal problem is ignored. This is 
because in a typical WLAN environment, every node can sense all the other transmissions, although it may not necessarily be able to correctly receive the packets from all other nodes [4].

Unlike wired network, the actual available bandwidth $B_{a v l}$ is less than the network average bandwidth $B_{\text {avg }}$ due to the wireless collision and backoff idle. If we define $T_{\text {suc }}$ as the average time period associated with successful transmission, $T_{c o l}$ as the average time period associated with packet collision and $T_{i d l e}$ as the average time period associated with backoff idle in a certain time interval. We can obtain

$$
B_{a v l}=\frac{T_{s u c}}{T_{\text {suc }}+T_{c o l}+T_{i d l e}} \times B_{a v g} .
$$

To calculate $T_{\text {suc }}, T_{\text {col }}$ and $T_{\text {idle }}$, we assume the total active number of full duplex VoIP calls in the network is n. To simplify the analysis and yet reveal the characteristics of the VoIP in IEEE 802.11 MAC protocol, we assume one full duplex VoIP call equals two half duplex connections established between active stations and Base Station (BS). Therefore, we can assume that the VoIP traffic is uniformly distributed among these $2 n$ active stations. If we assume the transmission probability for each active station in any time slot is $\tau$. We can obtain the following equtions according to [3], [6]

$$
\left\{\begin{array}{l}
p_{i}=(1-\tau)^{2 n} \\
p_{s}=2 n \tau(1-\tau)^{2 n-1} \\
p_{c}=1-p_{i}-p_{s}=1-(1-\tau)^{2 n}-2 n p(1-\tau)^{2 n-1}
\end{array}\right.
$$

where $p_{i}$ is the probability that the observed backoff time slot is idle, $p_{s}$ is the probability that there is one successful transmission, and $p_{c}$ is the collision probability that there are at least two concurrent transmissions at the same backoff time slot. Hence (1) becomes

$$
B_{a v l}=\frac{p_{s} T_{s}}{p_{s} T_{s}+p_{c} T_{c}+p_{i} T_{i}} \times B_{a v g},
$$

where $T_{s}$ is the time of a successful transmission, $T_{c}$ is the time wasted by a packet collision, and $T_{i}$ is the duration of an empty slot time. We know from [6]

$$
\begin{aligned}
& T_{s}=D I F S+D A T A+S I F S+A C K \\
& T_{c}=D A T A+E I F S \\
& T_{i}=\text { A Slot Time }
\end{aligned}
$$

where DATA is the time needed to transmit a data packet including IP header and MAC header, and all of these values are shown in Table I.

Here we do not use RTS/CTS mechanism, because the RTS and CTS frames are too large as shown in Table I compared with the payload data. Therefore using RTS/CTS mechanism will waste certain bandwidth.

The payload rate of voice data for each station is equal to $R_{\text {codec }}$ which represents the Bit Rate of codec. Although the $R_{\text {codec }}$ is very low (for example $R_{\text {codec }}$ of Codec G.729a which is the mostly used codec in VoIP applications is $8 \mathrm{kbps}$ ), the bandwidth required to transmit these data payload is very large. It is because that compared with the time period $T_{s}$
TABLE I

IEEE 802.11 SYSTEM PARAMETERS

\begin{tabular}{|c|c|}
\hline$R_{\text {data }}$ Bit rate for DATA packets & $2 \mathrm{Mbps}$ \\
\hline$R_{\text {basic }}$ Bit rate for RTS/CTS/ACK & $2 \mathrm{Mbps}$ \\
\hline$C W_{\min }$ & 31 \\
\hline$C W_{\max }$ & 1024 \\
\hline PLCPDataRate & $1 \mathrm{Mbps}$ \\
\hline A Slot Time & $20 \mu \mathrm{s}$ \\
\hline SIFS & $10 \mu \mathrm{s}$ \\
\hline DIFS & $50 \mu \mathrm{s}$ \\
\hline EIFS & $364 \mu \mathrm{s}$ \\
\hline MAC header & $224 \mathrm{bits}$ \\
\hline IP header & $160 \mathrm{bits}$ \\
\hline RTS & $160 \mathrm{bits}$ \\
\hline CTS, ACK & $112 \mathrm{bits}$ \\
\hline
\end{tabular}

which represents the time needed to send a packet successfully, the time to transmit the payload information $T_{p}$ is much shorter. As payload information is only a small part of data packet due to the overhead added in each layer as shown in Table I, and from Equation 4 we know that the time to send data is just a part of $T_{s}$. Therefore, we can obtain the required bandwidth

$$
B_{r e q}=\frac{T_{s}}{T_{p}} \times R_{c o d e c},
$$

where $T_{p}$ can be calculated by

$$
T_{p}=\text { Frame Size } \times \text { Frames Per Packet } / R_{\text {data }},
$$

where $R_{\text {data }}$ is the data rate of network shown in Table I, and Frame Size is the length of a frame coded by codec, for example, the Frame Size of Codec G.729a is 80 bits. Thus, we can obtain the maximum number of VoIP calls $\mathrm{N}$ as

$$
N=B_{a v l} /\left(2 \times B_{r e q}\right),
$$

Here, the factor 2 indicates that VoIP is a dual connections.

Bianchi in [6] provides us a group of equations to calculate $\tau$ in Equation (2) for a saturate network. In our cases, these equations become

$$
\begin{gathered}
\tau=\frac{2(1-2 p)}{(1-2 p)(W+1)+p W\left(1-(2 p)^{m}\right)}, \\
p=1-(1-\tau)^{2 n-1},
\end{gathered}
$$

where $p$ is referred as conditional collision probability which represents the probability that a collision occurs if a packet starts transmitting over the channel, $W$ is equal to $C W_{\min }$, $C W_{\max }$ is equal to $2^{m} W$ and $n$ is the number of VoIP calls in the network. If we obtain $\tau$, we can use Equations (2) and (3) to calculate $B_{a v l}$ of network when it comes into saturate mode. As shown in [4], the $B_{a v l}$ of a network in the saturate status is 0.9 times of the maximum $B_{a v l}$ a network can provide. 
Therefore, if we use Equations (10) and (11) to calculate $B_{a v l}$, the Equation (9) should be modified as

$$
N=\frac{B_{a v l} / 0.9}{2 \times B_{r e q}}
$$

We can simplify $\mathrm{N}$ as

$$
N=\frac{p_{s} T_{p}}{p_{s} T_{s}+p_{c} T_{c}+p_{i} T_{i}} \times \frac{B_{\text {avg }} / 0.9}{R_{\text {codec }} \times 2} .
$$

Thus, given the parameters of the codec and the network, if we let $\mathrm{n}$ equals to $\mathrm{N}$, we can obtain the maximum number of calls network can support by solving Equations (2), (10), (11) and (13).

Take Codec G.729a and network parameters shown in Table I as an example. For Codec G.729a, the bit rate of codec $R_{\text {codec }}$ is $8 \mathrm{kbps}$ ( $80 \mathrm{bits}$ for $10 \mathrm{~ms}$ frames). If we choose 2 frames per packet to transmit which represents $20 \mathrm{~ms}$ interval to transmit 20 bytes. Using Equations (4) (5) and (6), we can obtain that $T_{s}=50+464+10+248=772 \mu s, T_{c}=464+$ $364=828 \mu s, T_{p}=80 \mu s, T_{i}=20 \mu s$. Then, we can obtain the maximum number of calls network can support $\mathrm{N}$ is 10.4945 .

\section{ATICAC StRATEgy TO ENHANCE QOS OF VoIP IN 802.11 WLANS}

In this section, we will introduce adaptive transmitting interval call admission control (ATICAC) strategy to enhance the quality of VoIP calls in the network, aiming at preventing the network from saturation. The basic idea of ATICAC is that we find that increasing the transmitting interval of existing calls can decrease the network average collision probability $p_{c}$ which determines whether the network is saturate or not. In the meanwhile, the large transmitting interval may lead to large delay for the voice. Thus, ATICAC is to adaptively choose the transmitting interval according to the network status.

As we have discussed in the above section, if the network works in nonsaturate status, the quality of exiting VoIP in the network can be ensured. The main strategy of our call admission control is to prevent the network from saturation. Using our call admission control strategy, a new call will be accepted without highly reducing the quality of existing calls and the quality of existing calls will increase when a call leaves.

As we have discussed in the above section, if we can control the average collision probability $p_{c}$ of the network less than 0.1 , all the quality of existing calls will be guaranteed, which is the most important standard of our call admission control strategy. Here comes another problem that the average collision probability $p_{c}$ of the network is difficult to obtain because every station in the network including BS, does not know whether other nodes are in collision or not. Therefore, we use a new parameter called channel busyness probability as in [5] $p_{b}$ instead of $p_{c}$ which is the probability that a node senses the channel is busy. $p_{b}$ can be easily obtained by every station, since the IEEE 802.11 is a CSMA-based MAC protocol, working on the physical and virtual carrier sensing mechanisms. We will prove below that $p_{b}$ is relative with $p_{c}$ and BS can use $p_{b}$ to obtain $p_{c}$ which determines the state of the network.

Given the transmission probability for each active station in any time slot $\tau$ and Equation (2) we have discussed above, the channel busyness probability $p_{b}$ can be expressed as

$$
p_{b}=1-p_{i}=1-(1-\tau)^{2 n},
$$

where the number of connecting calls $n$ can be known by BS. Thus, combined Equation (2) and (14), $p_{c}$ can be expressed by $p_{b}$ as

$$
\left\{\begin{aligned}
p_{c} & =1-\left(1-p_{b}\right)-2 n \tau \frac{1-p_{b}}{1-\tau} \\
\tau & =1-\sqrt[2 n]{1-p_{b}}
\end{aligned}\right.
$$

Hence, BS can quickly obtain $p_{c}$ through $p_{b}$ and $n$ to administrate the network.

We can obtain from Equation (7) that the longer transmitting interval, the less required bandwidth $B_{r e q}$ is since the larger packet means the smaller overhead ratio, and less $B_{r e q}$ means more calls can be allowed in the network from Equation (9). But this is not the main reason which enlarges the number of calls in the network. Longer transmitting interval means fewer packets to transmit in the network, which results in less collisions in the network. Thus, the collisions probability of the network $p_{c}$ will be reduced and more VoIP calls can be allowed to come into the network.

The longer transmitting interval is, the better it will be due to the less required bandwidth and smaller collision probability in the network, while it is not suitable for VoIP transition since end-to-end delay of VoIP call is tightly restricted. We have obtained from subsection B of Section III that even there is no collision in the network, the end-to-end delay should be less than $244 \mathrm{~ms}$. If two users in separate WLANs build a VoIP call, the end-to-end delay between stations and BS should be less than $122 \mathrm{~ms}$ without considering wired internet delay. Longer transmitting interval means larger voice delay which is a part of end-to-end delay. Therefore, there is a trade off between the number of VoIP calls and end-to-end delay, i.e., the quality of VoIP calls.

Hence, we can describe our admission control strategy with adaptive transmitting intervals. When there is a new call arriving at the BS, the BS should calculate the network average collision probability $p_{c}$ through $p_{b}$, if $p_{c}$ is smaller than 0.1 , it means the network is not saturate, and the new call will be accepted; If not, it means the network comes into saturation, BS will decide whether to decrease the quality of existing calls by enlarging the transmitting interval of all calls including the new one or not. If the quality of existing calls is poor, i.e., the transmitting interval is $50 \mathrm{~ms}$, the new call should be rejected by BS. Otherwise, BS will enlarge the transmitting interval of all calls including the new one to accept the new call. In another case, when a existing call finishes, the BS will attempt to diminish the transmitting interval of the rest existing calls to enhance the quality of these calls, and then calculate $p_{c}$ again. If $p_{c}$ is smaller than 0.1 , the attempt is successful, and all the 
existing calls will have better quality; If not, the attempt is fail, all the existing calls will operate in previous transmitting interval, and the quality of calls will not be enhanced much.

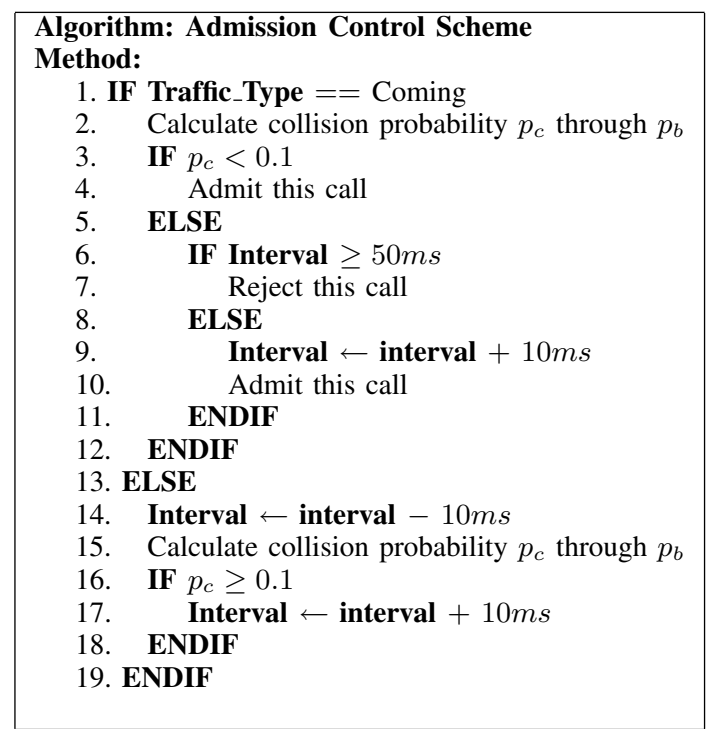

Fig. 1. Admission Control Implementation.

\section{Model Validation And Performance Evaluation}

In this section, we evaluate our ATICAC through extensive $n s-2$ [7] simulations. We verify the analytical results for VoIP in WLAN in Section III. Further we show that our call admission control strategy with adaptive transmitting interval in Section IV can improve the performance of VoIP over IEEE 802.11 WLAN in terms of more admitted calls, smaller delay, and almost zero packet loss rate.

\section{A. Simulation Environments}

In our simulation study, the radio propagation model is TwoRay Ground model and the a wireless LAN using IEEE 802.11 MAC protocol with direct sequence spread spectrum (DSSS). Other defaults parameters are summarized in Table I. We use different numbers of mobile stations in a rectangular grid with dimension $150 \times 150 \mathrm{~m}^{2}$ to simulate the Wireless LAN. We use CBR traffic to simulate half duplex VoIP connections.

\section{B. Model Validation}

Figure 2 shows the maximum number of calls with transmitting interval increasing, using different $C W_{\min }$ and data rate of the network, to compare our analytical model with simulation results. We obtain the analytical results using the analytical model for VoIP in 802.11 WLAN in Equation (2), (10), (11) and (13) compared with $n s-2$ simulation results. We can see that the analytical results closely match the

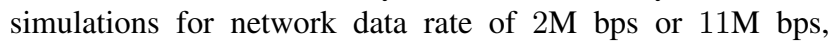
with varieties of $C W_{\min }=15,31,63$. The maximum number of call supported increases with the increase of transmitting interval. We can also find that the $C W_{\min }$ takes a little effect on the maximum number of calls supported in the network because larger $C W_{\text {min }}$ provides more backoff timer selections. Therefore, it can decrease the average collision probability at certain range. In our analytical model and simulation results, increasing $C W_{\text {min }}$ from 15 to 63 can allow one more call to join the network at most in some situations. This suggests that our analytical model is robust against $C W_{\min }$.

\section{Performance Evaluation}

To evaluate the performance of ATICAC, we consider a scenario that VoIP calls join the network one by one every 2 seconds from 102 second. All the calls in the simulation use fixed $20 \mathrm{~ms}$ transmitting interval which is wildly used for VoIP calls except for ATICAC. In Figure 3, the line with legend star represents the network performance when there is no call admission control. After the eleventh call joins the network at the time 122 second, the network falls into saturate mode, the average throughput decreases slightly and the average delay and average loss rate increase dramatically in Figure 3-(b) and (c). The line with legend triangle represents the network performance using normal call admission control. When the number of existing calls in the network attains the maximum value, i.e., 10 calls, the base station will reject new arriving calls until one existing call finishes. The normal call admission control strategy can guarantee the quality of existing calls, while the number of ongoing calls is still small and the capacity of WLAN for VoIP is not fully explored. Compared with normal CAC strategy, our ATICAC strategy (with legend cross) allows twice simultaneous calls as that of the normal CAC strategy, i.e., $100 \%$ improvement, at the expense of 30 $\mathrm{ms}$ delay increase. We note that the increase of $30 \mathrm{~ms}$ for delay incurs 0.72 decrease of R-score, which is still in the tolerable range of VoIP call as we have discussed in Section III. On the other hand, if there are small number of calls in the network, for example, less than six calls in the network, the average delay of our ATICAC strategy is smaller than normal CAC strategy due to smaller transmitting interval which adaptively decreases.

\section{CONCLUSION}

In this paper, we study how IEEE 802.11 WLANs can support more VoIP calls, and we provide a simple analytical model, which is able to calculate the maximum number of VoIP calls supported in IEEE 802.11 WLANs accurately compared with simulation results. Using the proposed model, we find that the high collision probability takes the main responsibility to the decrease of Qos of VoIP calls if too many calls join the network. We propose a new call admission control strategy, adaptive transmitting interval call admission control (ATICAC). In the ATICAC, BS control the average collision probability $p_{c}$ of the network to prevent the network from saturation. BS will adaptively change the transmitting interval of active stations when a call arrives or leaves. The ATICAC can ensure the QoS of VoIP calls in 802.11 WLANS, and at the same time provide more VoIP calls for a given WLAN. We also evaluate the performance of ATICAC through 


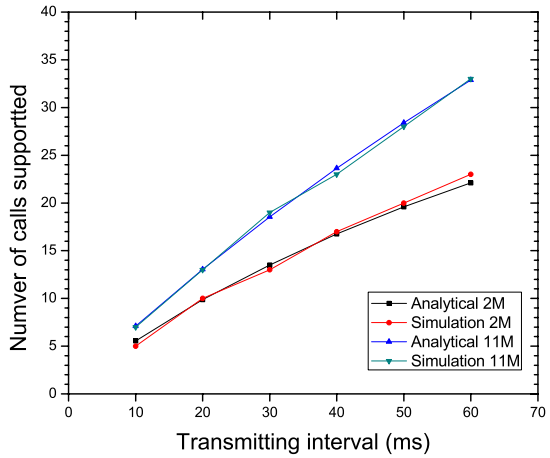

(a) $C W_{\min }=15$

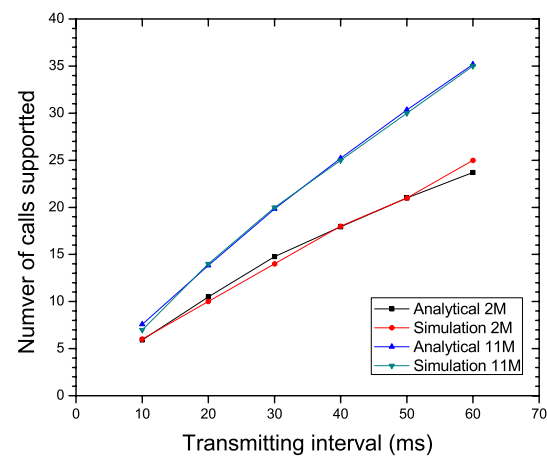

(b) $C W_{\min }=31$

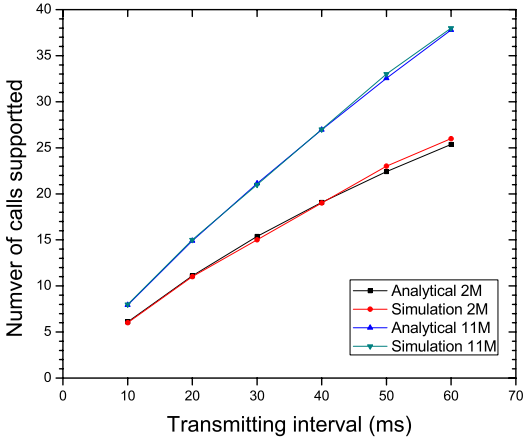

(c) $C W_{\text {min }}=63$

Fig. 2. Maximum number of calls supported with transmitting interval increasing.

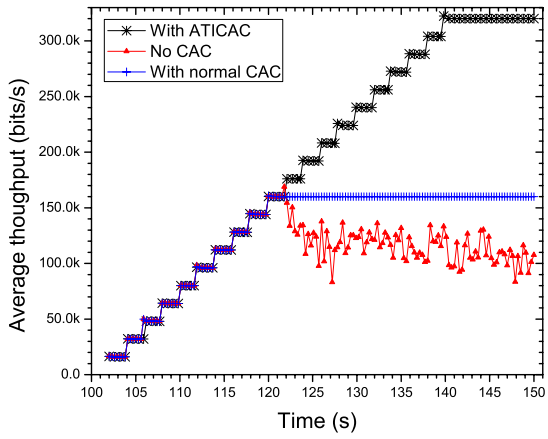

(a) Average throughput of network

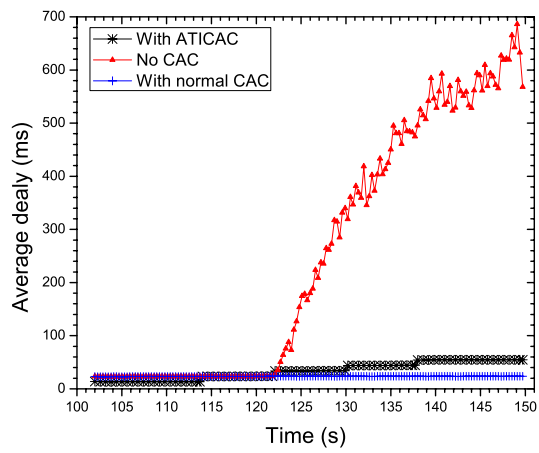

(b) Average delay of calls

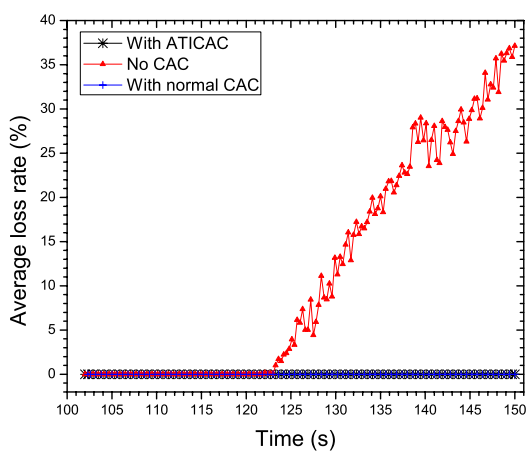

(c) Average loss rate of calls

Fig. 3. Call quality statistics with the number of calls increasing.

extensive $n s-2$ simulations. The results indicate that ATICAC can provide $50-100 \%$ more VoIP calls according to different scenarios. As to the same number of calls, ATICAC provides up to $50 \%$ less delay than the normal CAC strategy.

\section{ACKNOWLEDGMENT}

This work is supported by NSF China (No. 60702046); China Ministration of Education (No. 20070248095); Shanghai Jiaotong University Young Faculty Funding; Shanghai Jiaotong University Pre-Research Funding; Qualcom China Research Grant. NSF China \#60672067.

\section{REFERENCES}

[1] H. Wei, K. Kim, A. Kashyap and S. Ganguly, "On Admission of VoIP Calls Over Wireless Mesh Network," In Proceedings of ICC 2006.

[2] S. Garg and M. Kappes, "Can I add a VoIP call?," in IEEE International Conference on Communications(ICC), (Anchorage, Alaska), 2003.

[3] IEEE Standard for Wireless LAN Medium Access Control (MAC) and Physical Layer (PHY) Specifications, ISO/IEC 8802-11: 1999(E), 1999.

[4] H. Zhai, X. Chen and Y. Fang "How Well Can the IEEE 802.11 Wireles LAN Support Quality of Service?" in IEEE Transactions on Wireless Communications, vol. 4, no. 6, november 2005

[5] H. Zhai, X. Chen and Y. Fang "A Call Admission and Rate Control Scheme for Multimedia Support over IEEE 802.11Wireless LANs," In Proceedings of the First International Conference on Quality of Service in Heterogeneous Wired/Wireless Networks (QSHINE04)
[6] G. Bianchi, "Performance analysis of the IEEE 802.11 distributed coordination function,” IEEE J. Sel. Areas Commun. vol. 18, no. 3, pp. 535-547, Mar. 2000.

[7] NS-2 Simulator http://www.isi.edu/nsnam/ns/

[8] C. Li et al., "An Adaptive IEEE 802.11 Scheme for Voice and Data Services in Wireless LANs," in Proc. Fifth Annual Conference on Communication Networks and Services Research(CNSR'07)

[9] S. Choi et al., "IEEE 802.11e contentionbased channel access (EDCF) performance evaluation," in Proc. IEEE Int. Conf. Communications (ICC), Anchorage, AK, 2003, pp. 1151-1156.

[10] Draft Supplement to Part 11: Medium Access Control (MAC) Enhancements for Quality of Service (QoS), IEEE Std 802.11e/D8.0, Feb. 2004

[11] Mo Li and Yunhao Liu, "Rendered Path: Range-Free Localization in Anisotropic Sensor Networks with Holes", ACM MobiCom 2007, Montreal, Quebec, Canada, September 2007

[12] Mo Li and Yunhao Liu, "Underground Structure Monitoring with Wireless Sensor Networks", ACM/IEEE IPSN, Cambridge, Massachusetts, USA, April, 2007

[13] J. Yu, S. Choi, and J. Lee, "Enhancement of voip over ieee 802.11 wlan via dual queue strategy," in In Proceedings of ICC 2004.

[14] W.Wang, S. Liew, and V. Li, "Solutions to performance problems in voip over a 802.11 wireless lan,” in In IEEE Trans. on Vehicular Technology, vol. 54, Jan 2005

[15] C. Li, J. Li, and X. Cai, "A novel self-adaptive transmission scheme over an IEEE 802.11 WLAN for supporting multi-service," Wireless Communications and Mobile Computing, vol. 6, no. 4, Jun. 2006, pp. 467-474.

[16] S. Shin, H. Schulzrinne, "Experimental Measurement of the Capacity for VoIP Traffic in IEEE 802.11 WLANs," in Preceeding of INFOCOM 2007. 